\title{
Asymmetric Landau-Zener tunneling in a periodic potential
}

\author{
M. Jona-Lasinio, O. Morsch, M. Cristiani, N. Malossi, J.H. Müller, E. Courtade, M. Anderlini and E. Arimondo \\ INFM, Dipartimento di Fisica E.Fermi, Università di Pisa, Via Buonarroti 2, I-56127 Pisa,Italy
}

(Dated: November 13, 2018)

\begin{abstract}
Using a simple model for nonlinear Landau-Zener tunneling between two energy bands of a BoseEinstein condensate in a periodic potential, we find that the tunneling rates for the two directions of tunneling are not the same. Tunneling from the ground state to the excited state is enhanced by the nonlinearity, whereas in the opposite direction it is suppressed. These findings are confirmed by numerical simulations of the condensate dynamics. Measuring the tunneling rates for a condensate of rubidium atoms in an optical lattice, we have found experimental evidence for this asymmetry.
\end{abstract}

PACS numbers: PACS number(s): 03.65.Xp, 03.75.Lm

The phenomenon of Landau-Zener (LZ) tunneling 1 ] is a basic quantum mechanical process. It is based on the solution of the Schrödinger equation for a two-level dynamics when a parameter of the Hamiltonian system is time dependent. If at time $t=-\infty$ the system is prepared in one adiabatic state of the Hamiltonian, the time dependence of the Hamiltonian implies that at time $t=+\infty$ there is a finite probability that the system will occupy the other adiabatic state. As far as this tunneling behaviour is concerned, complete symmetry exists between the adiabatic states. Variations of the LZ model have been studied 2, 3, 4], and an observation of LZ dynamics in classical optical systems has been reported [5]. More recently, LZ tunneling within a periodic potential was studied for a nonlinear two-level system in which the level energies depend on the occupation of the levels [6, 7]. It was discovered that a nonlinearity with a positive sign enhances the tunneling probability between the ground band and the first excited band. Moreover, Niu and coworkers discovered a nonzero LZ tunneling probability even in the fully adiabatic limit when the nonlinearity was larger than a critical value [7]. Critical values for deformations of the energy level structures were obtained in refs. [6, 8, 9]. In a Bose-Einstein condensate inside a periodic potential such as an optical lattice, the mean-field interaction between the atoms can be comparable to other energy scales of the system and hence the level-dependent energy shift can lead to an observable modification of the tunneling behaviour.

In the present work we explore, theoretically and experimentally, the Landau-Zener tunneling between Bloch bands of a Bose-Einstein condensate in an accelerated optical lattice. The optical lattice depth controls the tunneling barrier, while the optical lattice acceleration controls the time dependence of the Hamiltonian. We show that the mean-field nonlinearity produces an asymmetry for the tunneling probability between the adiabatic states of the Hamiltonian. More precisely the tunneling probability from the lower energy adiabatic state to the upper one is enhanced, while the tunneling probability from the higher energy level to a lower level is suppressed. Numerical integration of the one-dimensional Gross-Pitaevskii equation and a simple two-state model demonstrate this asymmetry. Moreover, our experimental data obtained with a rubidium Bose-Einstein condensate confirms this prediction.

The qualitative explanation of this asymmetry in the tunneling transition probabilities depends on the choice of the quantum representation used for the description of the nonlinear system. We have identified two different basic mechanisms, acting simultaneously or independently. In the first mechanism the nonlinear term of the Schrödinger equation acts as a perturbation whose strength is proportional to the energy level occupation. If the initial state of the condensate in the lattice corresponds to a filled lower level of the state model, then the lower level is shifted upward in energy while the upper level is left unaffected. This reduces the energy gap between the lower and upper level and enhances the tunneling. On the contrary, if all atoms fill the upper level then the energy of the upper level is increased while the lower level remains unaffected. This enhances the energy gap and reduces the tunneling. The overall balance leads to an asymmetry between the two tunneling processes. In a different representation the nonlinearity term in the Schrödinger equation produces an additional term to the optical lattice depth. This additional term increases or decreases the optical lattice depth depending on whether the tunneling process proceeds from the lower level to the upper one, or vice versa. Thus the tunneling due to a weaker or stronger potential barrier is increased or decreased, as the case may be, depending on the initial state.

The motion of a Bose-Einstein condensate in an accelerated 1D optical lattice is described by the GrossPitaevskii equation

$$
\begin{aligned}
i \hbar \frac{\partial \psi}{\partial t}=\frac{1}{2 M}( & \left.-i \hbar \frac{\partial}{\partial x}-M a_{L} t\right)^{2} \psi+ \\
& +\frac{V_{0}}{2} \cos \left(2 k_{l} x\right) \psi+\frac{4 \pi \hbar^{2} a_{s}}{M}|\psi|^{2} \psi
\end{aligned}
$$

where $M$ is the atomic mass, $k_{L}=\pi / d$ is the optical lattice wavenumber with $d$ the optical lattice step, and $V_{0}$ is the strength of the periodic potential depth. The $s$-wave scattering length $a_{s}$ determines the nonlinearity of the system. Equation 1 is written in the comoving frame of the lattice, so the inertial force $M a_{L}$ appears as 
a momentum modification. The wavefunction $\psi$ is normalized to the total number of atoms in the condensate and we define $n_{0}$ as the average uniform atomic density. Defining the dimensionless quantities $E_{\text {rec }}=\hbar^{2} k_{L}^{2} / 2 M$, $\tilde{x}=2 k_{L} x, \tilde{t}=8 E_{\text {rec }} t / \hbar$, and rewriting $\tilde{\psi}=\psi / \sqrt{n_{0}}$, $\tilde{v}=V_{0} / 16 E_{r e c}, \tilde{\alpha}=M a_{L} / 16 E_{r e c} k_{L}, C=\pi a_{s} n_{0} / k_{L}^{2}$, Eq. (11) is cast in the following form [7]:

$$
i \frac{\partial \psi}{\partial t}=\frac{1}{2}\left(-i \frac{\partial}{\partial x}-\alpha t\right)^{2} \psi+v \cos (x) \psi+C|\psi|^{2} \psi
$$

where we have replaced $\tilde{x}$ with $x$, etc. In the neighborhood of the Brillouin zone edge we can approximate the wave function by a superposition of two plane waves (the two level model of ref. 7]), assuming that only the ground state and the first excited state are populated. We then substitute $\psi(x, t)=a(t) e^{i q x}+b(t) e^{i(q-1) x}$, with $|a(t)|^{2}+|b(t)|^{2}=1$ in Eq. (2). Comparing the coefficients of $e^{i q x}$ and $e^{i(q-1) x}$, linearizing the kinetic terms and dropping the irrelevant constant energy $1 / 8+C\left[1+\left(|a|^{2}+|b|^{2}\right) / 2\right]$, Eq. (2) assumes the form

$$
i \frac{\partial}{\partial t}\left(\begin{array}{l}
a \\
b
\end{array}\right)=\left[\frac{\alpha t}{2} \sigma_{3}+\frac{v}{2} \sigma_{1}\right]\left(\begin{array}{l}
a \\
b
\end{array}\right)+\frac{C}{2}\left(|b|^{2}-|a|^{2}\right) \sigma_{3}\left(\begin{array}{l}
a \\
b
\end{array}\right)
$$

where $\sigma_{i} i=1,2,3$ are the Pauli matrices [10]. The adiabatic energies of Eq. 3 have a butterfly structure at the band edge of the Brillouin zone for $C \geq v[7,8,9]$, but in the present work we always work in a regime where $C \ll v$, hence that structure plays no role.

In the linear regime $(C=0)$, evaluating the transition probability in the adiabatic approximation, we find the linear LZ formula for the tunneling probability $r$

$$
r=e^{-\frac{\pi v^{2}}{2 \alpha}}
$$

expressing the occupation changes in terms of the rate $\alpha$ at which the diagonal energies of the linear Hamiltonian change their value, and of the off-diagonal interaction energy $v$. In the nonlinear regime, as the nonlinear parameter $C$ grows, the lower to upper tunneling probability grows as well until an adiabaticity breakdown occurs at $C=v[7]$. The upper to lower tunneling probability, on the other hand, decreases with increasing nonlinearity [11]. We derived the tunneling rate from the numerical integration of Eq. (3). In Fig. 1(a) we plot the lower to upper tunneling rate (initial $(a, b)=(1,0))$ and the upper to lower tunneling rate (initial $(a, b)=(0,1)$ ) of the Bose-Einstein condensate as a function of the nonlinear parameter $C$ for different accelerations of the optical lattice. We see that for $C=0$ the rate is the same for both tunneling directions whereas for $C \neq 0$ the two rates are different, and the smaller the acceleration the larger the difference. This result is intuitive since for very small accelerations the main contribution originates from the nonlinear effect (the linear tunneling of Eq. (4) being small) while for large accelerations the main contribution comes from the linear effect. We confirmed the presence of a tunneling asymmetry by integrating directly Eq. (10) (taking into account the full experimental protocol described below), finding qualitative agreement with the prediction of the two-state model. For small $C$ values, we have fitted the $C$ dependence of the tunneling rate of Fig 1 (a) through the following expression [6, 7]]:

$$
r(C)=e^{-\frac{\pi v^{2}}{2 \alpha}\left(1 \pm \beta \frac{C}{v}\right)}
$$

with $\beta=0.75,0.17,0.14$ for the different acceleration values.
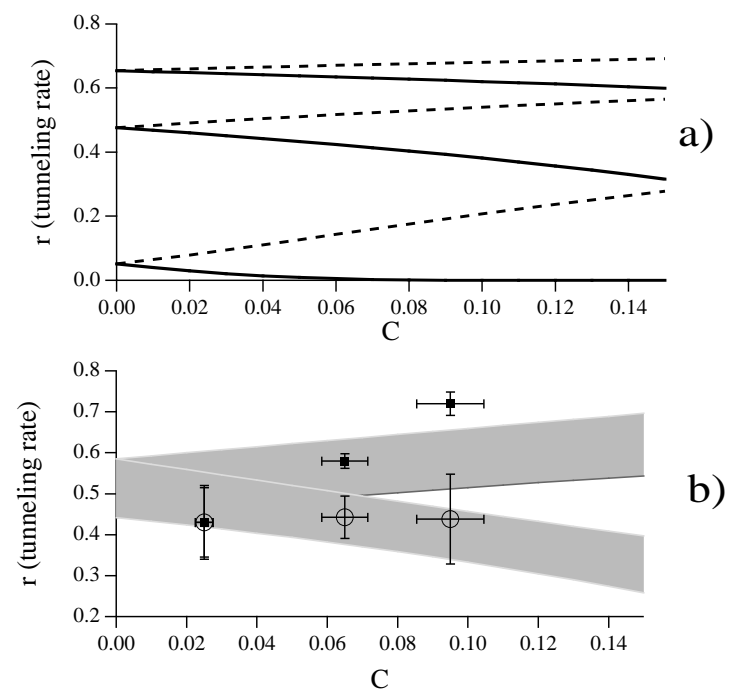

FIG. 1: LZ tunneling rate $r$ within the two-level model as a function of the nonlinear parameter $C$ for different accelerations. The rates coincide for $C=0$, whereas for $C \neq 0$ they differ significantly. Results are for $v=0.134$ corresponding to $V_{0}=2.2 E_{r e c}$, in (a) for $\alpha=0.01,0.04,0.07$, corresponding to $a_{L}=0.8,3.2,5.6 \mathrm{~m} \mathrm{~s}^{-2}$ from bottom to top and in (b) for $\alpha=0.036$ corresponding to $a_{L}=2.9 \mathrm{~ms}^{-2}$. In (b) experimental results are denoted by open symbols for the excited band to ground state band tunneling and by filled symbols for the ground state band to excited band tunneling. The experimental points at $C=0.025$ have been re-scaled from Fig. 3 using the Landau-Zener formula to match the acceleration to the one used to obtain the other experimental points. The shaded area represents the confidence region for the prediction of the two-level model taking into account the uncertainty in our measurement of the lattice depth.

The nonlinear regime is more easily interpreted by writing Eq. ([3]) as

$$
i \frac{\partial}{\partial t}\left(\begin{array}{l}
a \\
b
\end{array}\right)=\left[\frac{\alpha t}{2} \sigma_{3}+\frac{v}{2} \sigma_{1}\right]\left(\begin{array}{l}
a \\
b
\end{array}\right)-\frac{C}{2}\left(\begin{array}{cc}
|a|^{2} & -b^{*} a \\
-a^{*} b & |b|^{2}
\end{array}\right)\left(\begin{array}{l}
a \\
b
\end{array}\right)
$$

Here the nonlinear diagonal terms represent a shift of the energy levels, different for an occupation of the ground or upper level, decreasing or increasing the energy gap 
depending on the initial state. The off-diagonal terms of Eq. [6 produce an equivalent contribution to the nonlinear tunneling process. The off-diagonal terms modify the interaction term $v$ equivalent to a Rabi frequency in the two-level model. The off-diagonal scalar product between the two states $a^{*} b$, calculated applying the adiabatic approximation technique of [12], changes sign depending on the initial state (lower or upper). Thus the linear Rabi frequency is modified by the nonlinear offdiagonal term, and the increase or decrease depends on the initial state. This nonlinear modification of the interaction energy constitutes an additional contribution to the asymmetric tunneling rate.

Experimentally, we investigated the phenomenon of asymmetric tunneling between the energy bands of BoseEinstein condensates in an optical lattice using a setup described in detail in [13, 14]. Briefly, we create condensates of $N \approx 10^{4}$ rubidium atoms in a time-orbiting potential (TOP) trap. Once condensation has been achieved, the mean trapping frequency $\bar{\nu}_{\text {trap }}$ of the magnetic trap is adiabatically reduced to values between $15 \mathrm{~Hz}$ and $50 \mathrm{~Hz}$. Thereafter, two laser beams with waists of $1.8 \mathrm{~mm}$ and intersecting at an angle $\theta=38 \mathrm{deg}$ at the position of the condensate are switched on with a linear ramp of duration $\tau_{\text {ramp }}=10 \mathrm{~ms}$, thus ensuring adiabaticity of the loading process. The beams are detuned to the red side of the rubidium atomic resonance by $\approx 30 \mathrm{GHz}$ and have a variable frequency difference $\Delta \nu$ between them, controllable through two acousto-optic modulators which are also used to vary the intensity of the beams. In this way, a periodic potential with lattice constant $d=1.18 \mu \mathrm{m}$ and lattice recoil energy $E_{r e c} / h=455 \mathrm{~Hz}$ is created, which through the frequency difference $\Delta \nu$ can be made to move at a constant velocity $v=d \Delta \nu$ or accelerated with $a_{L}=d \frac{d \Delta \nu}{d t}$. For the current experiment, lattice depths between $0.25 E_{r e c}$ and $2.5 E_{r e c}$ were used.

Landau-Zener tunneling between the two lowest energy bands of a condensate inside an optical lattice is investigated in the following way. Initially, the condensate is loaded adiabatically into one of the two bands. Subsequently, the lattice is accelerated in such a way that the condensate crosses the edge of the Brillouin zone once, resulting in a finite probability for tunneling into the other band (higher-lying bands can be safely neglected as their energy separation at the edge of the Brillouin zone is much larger than the band gap). After the tunneling event, the two bands have populations reflecting the Landau-Zener tunneling rate (assuming that, initially, the condensate populated one band exclusively). In order to experimentally determine the number of atoms in the two bands, we then increase the lattice depth (from $\approx 2 E_{r e c}$ to $\approx 4 E_{r e c}$ ) and decrease the acceleration (from $\approx 3 \mathrm{~m} \mathrm{~s}^{-2}$ to $\approx 2 \mathrm{~m} \mathrm{~s}^{-2}$ ). In this way, successive crossings of the band edge will result in a much reduced Landau-Zener tunneling probability between the ground state band and the first excited band (of order a few percent). The fraction of the condensate that after the first tunneling event populated the ground state band will, therefore, remain in that band, whereas the population of the first excited band will undergo tunneling to the second excited band with a large probability (around 90 percent) as the gap between these two bands is smaller than the gap between the two lowest bands by a factor $\approx 5$ for our parameters. Once the atoms have tunneled into the second excited band, they essentially behave as free particles since higher-lying band-gaps are smaller still, meaning that the fraction of the condensate that populated the first excited band after the first tunneling event will no longer be accelerated by the lattice. In summary, using this experimental sequence we selectively accelerate that part of the condensate further that populates the ground state band. In practice, in order to get a good separation between the two condensate parts after a time-of-flight, we accelerate the lattice to a final velocity of $4-6 v_{\text {rec }}[15$ and absorptively image the condensate after $22 \mathrm{~ms}$ (see Fig. (2).
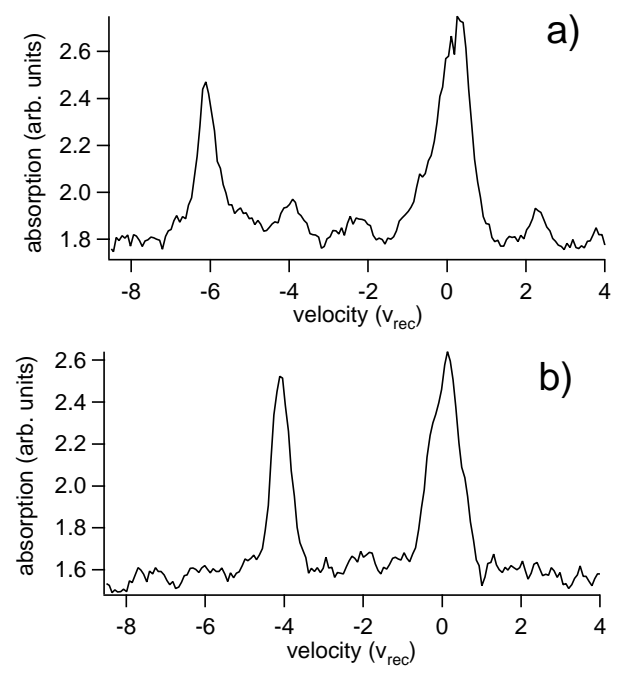

FIG. 2: Profiles of absorption images taken after $22 \mathrm{~ms}$ of time-of-flight of condensates released after the acceleration procedure described in the text. The condensates were prepared in the ground state band in (a) and in the first excited band in (b) within an optical lattice with depth $V_{0}=2.6 E_{r e c}$ and $a_{L}=2.9 \mathrm{~ms}^{-2}$.

In order to investigate tunneling from the ground state band to the first excited band, we adiabatically ramped up the lattice depth with the lattice at rest and then started the acceleration sequence. The tunneling from the first excited to the ground-state band is investigated in a similar way, except that in this case we initially prepare the condensate in the first excited band by moving the lattice with a velocity of $1.5 v_{\text {rec }}$ (through the frequency difference $\Delta \nu$ between the acousto-optic modulators) when switching it on. In this way, in order to conserve energy and momentum the condensate must populate the first excited band at a quasi-momentum half-way between zero and the edge of the first Brillouin zone 16 . Thereafter, the same acceleration sequence as described 
above is used in order first to induce Landau-Zener tunneling and then to separate the fraction of the condensate that underwent tunneling to the lowest band from the one that remained in the first excited band. For both tunneling directions, the tunneling rate is measured as

$$
r=\frac{N_{\text {tunnel }}}{N_{\text {tot }}}
$$

where $N_{\text {tot }}$ is the total number of atoms measured from the absorption picture. For the tunneling from the first excited band to the ground band, $N_{\text {tunnel }}$ is the number of atoms accelerated by the lattice, i.e. those detected in the final velocity class $4 v_{r e c}$, whereas for the inverse tunneling direction, $N_{\text {tunnel }}$ is the number of atoms detected in the $v=0$ velocity class. To illustrate our method

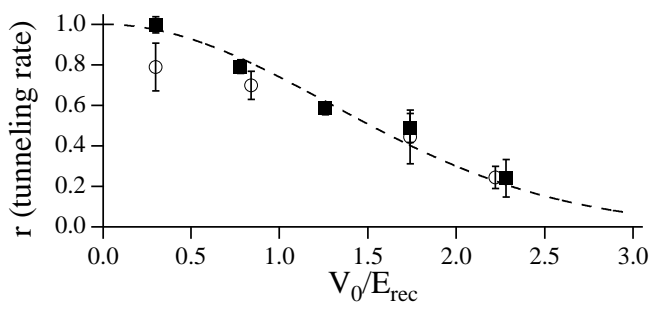

FIG. 3: Landau-Zener tunneling between the two lowest energy bands of a condensate in an optical lattice as a function of the lattice depth (carrying a $\pm 10 \%$ systematic error) for $\alpha=0.025$ corresponding to $a_{L}=2.0 \mathrm{~ms}^{-2}$. Tunneling rates from the ground state band to the first excited band (filled symbols) and vice versa (open symbols) are virtually identical and agree with the linear prediction (dashed line) as the nonlinear parameter $C \approx 0.025$ is small for the trap used in these measurements $\left(\bar{\nu}_{\text {trap }}=15 \mathrm{~Hz}\right)$. The deviation from theory of the filled symbol for small lattice depths is most likely due to a breakdown of the adiabatic approximation of the linear Landau-Zener model.

for measuring the tunneling in both directions, in Fig. [3 we report the two tunneling rates as a function of lattice depth for a condensate in a weak magnetic trap and hence a small value of the interaction parameter $C[17$.
In this case, both tunneling rates are essentially the same and agree well with the linear Landau-Zener prediction. By contrast, when $C$ is increased, the two tunneling rates begin to differ, as can be seen in Fig. 1(b). Qualitatively we find agreement with the theoretical predictions of the non-linear Landau-Zener model, whereas quantitatively there are significant deviations. We believe these to be partly due to experimental imperfections. In particular, the sloshing (dipolar oscillations) of the condensate inside the magnetic trap can lead to the condensate not being prepared purely in one band due to non-adiabatic mixing of the bands if the initial quasimomentum is too close to a band-gap. Furthermore, a numerical simulation of the experiment shows that for large values of $C$, for which the magnetic trap frequency was large, the measured tunneling rates are significantly modified by the presence of the trap. We have, however, verified that when $C$ in the simulation is varied without varying the trap frequency, the asymmetric tunneling effect persists.

In summary, we have numerically simulated LandauZener tunneling between two energy bands in a periodic potential and found that, in the presence of a nonlinear interaction term, an asymmetry in the tunneling rates arises. Experimentally, we have measured these tunneling rates for different values of the interaction parameter and found qualitative agreement with the simulations. We note here that the phenomenon of asymmetric tunneling should be a rather general feature of quantum systems exhibiting a nonlinearity. For instance, calculating the energy shift due to a nonlinearity for two adjacent levels of a harmonic oscillator, one finds that both levels are shifted upwards in energy, the shift being proportional to the population of the respective level. The energy difference between the levels, therefore, decreases if only the lower state is populated and increases if all the population is in the upper level. Finally, we note that statedependent mean-field shifts have also been observed in measurements of the clock-shift in ultra-cold and Bosecondensed atomic samples [19.

This work was supported by the MURST (COFIN2000 Initiative), the INFM (PRA 'Photonmatter'), and by the EU through Contract No. HPRN-CT-2000-00125.
[1] L.D. Landau, Phys. Z. Sowjetunion, 2, 46 (1932); G. Zener, Proc. R. Soc. London, Ser. A, 137, 696 (1932).

[2] K. Mullen, E. Ben-Jacob, Y. Gefen and Z. Schuss, Phys. Rev. Lett. 62, 2543 (1989).

[3] Y. Gefen, E. Ben-Jacob, and A.O. Caldeira, Phys. Rev. B 36, 2770 (1987).

[4] S. Fishman, K. Mullen, and E. Ben-Jacob, Phys. Rev. A 42, 5181 (1990).

[5] D. Bouwmeester, N.H. Dekker, F.E.v. Dorsselaer, C.A. Schrama, P.M. Visser and J.P. Woerdman, Phys. Rev. A 51, 646 (1995).

[6] O. Zobay and B.M. Garraway, Phys. Rev. A 61, 033603 (2000).
[7] B. Wu and Q. Niu, Phys. Rev. A 61, 023402 (2000); J. Liu et al., ibidem 66, 023404 (2002).

[8] D. Diakonov, L.M. Jensen, C.J. Pethick, and H. Smith, Phys. Rev. A 66, 013604 (2002); M. Machholm, C.J. Pethick, and H. Smith, Phys. Rev. A 67, 053613 (2003).

[9] E.J. Mueller, Phys. Rev. A 66, 063603 (2002).

[10] An equivalent equation is obtained using wavefunctions $\exp (i k x) \cos (k x)$ and $\exp (i k x) \sin (k x)$ describing the eigenstates at the band edge.

[11] For occupation with equal amplitude of the lower and upper states $|a|^{2}=|b|^{2}$, the nonlinear corrections leading to the asymmetric tunneling play no role. 
[12] M.D. Crisp, Phys. Rev. A 8, 2128 (1973).

[13] O. Morsch et al., Phys. Rev. Lett. 87, 140402 (2001).

[14] M. Cristiani et al., Phys. Rev. A 65, 063612 (2002).

[15] In order to keep the total interaction time with the lattice constant, the final velocity of the lattice when the condensate is prepared in the upper state is $\approx 4 v_{r e c}$ since initially the lattice is moving at $-1.5 v_{\text {rec }}$.

[16] E. Peik et al., Appl. Phys. B 65, 685 (1997).

[17] For the non-uniform condensate density in the optical lattice, described by Pedri et al. [18] with a separation of the variables parallel and orthogonal to the optical latttice, the parameter $C$ of the 1D Eq. 1 was determined from the maximum condensate density.

[18] P. Pedri et al., Phys. Rev. Lett. 87, 220401 (2001).

[19] D.M. Harber, H.J. Lewandowski, J.M. McGuirk, and E.A. Cornell, Phys. Rev. A 66, 053616 (2002) and references therein. 